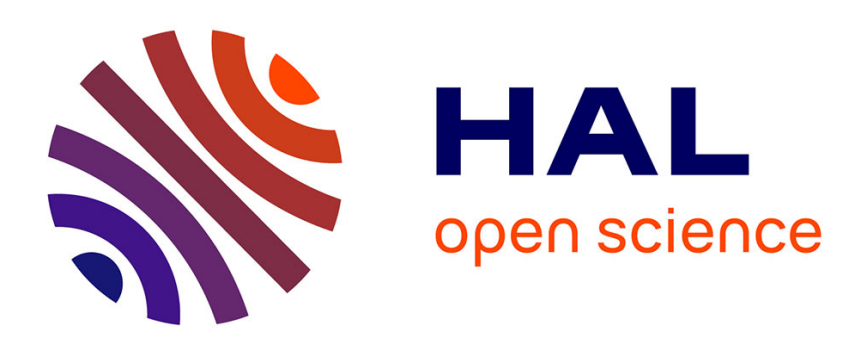

\title{
Les effets photovoltaiques et photorésistants dans les composés II-VI en couches minces
}

\author{
M. Balkanski, B. Choné
}

\section{To cite this version:}

M. Balkanski, B. Choné. Les effets photovoltaiques et photorésistants dans les composés II-VI en couches minces. Revue de Physique Appliquée, 1966, 1 (3), pp.179-188. 10.1051/rphysap:0196600103017900 . jpa-00242712

HAL Id: jpa-00242712

https://hal.science/jpa-00242712

Submitted on 1 Jan 1966

HAL is a multi-disciplinary open access archive for the deposit and dissemination of scientific research documents, whether they are published or not. The documents may come from teaching and research institutions in France or abroad, or from public or private research centers.
L'archive ouverte pluridisciplinaire HAL, est destinée au dépôt et à la diffusion de documents scientifiques de niveau recherche, publiés ou non, émanant des établissements d'enseignement et de recherche français ou étrangers, des laboratoires publics ou privés. 


\title{
LES EFFETS PHOTOVOLTAIQUES ET PHOTORÉSISTANTS DANS LES COMPOSÉS II-VI EN COUCHES MINCES
}

\author{
Par M. BALKANSKI \\ Laboratoire de Physique des Solides, Faculté des Sciences, Paris. \\ B. CHONÉ, \\ Bureau d'Analyse et de Recherche Appliquées
}

\begin{abstract}
Résumé. - Dans le sulfure de cadmium la photoconductivité s'explique par l'existence de plusieurs centres de recombinaison de diverses sections efficaces de capture, dus à la présence d'un léger excès de cadmium ou d'impuretés étrangères. L'effet photovoltaïque observé dans les photopiles en couches minces de CdS s'interprète comme conséquence d'une hétérojonction à l'interface de deux semiconducteurs différents. L'élaboration et les caractéristiques des photopiles sont décrites.
\end{abstract}

Abstract. - The photoconductivity in cadmium sulphide can be explained through the existence of several recombination-centers of different capture-cross-sections, caused by the presence of a slight excess of cadmium or other impurities. The photovoltaic effect observed in CdS film photocells is interpreted as a consequence of a hetero-junction at the interface of two differents semiconductors. Production and characteristics of the devices are described.

1. Introduction. - La photoconductivité et l'effet photovoltaïque dans les composés II-VI, et en particulier dans le CdS et le CdTe, ont fait l'objet de nombreuses études menées dans la plupart des cas sur des monocristaux. L'amélioration considérable, ces dernières années, des techniques d'évaporation a permis de mettre au point d'une façon systématique et contrôlée la préparation de couches minces de ces composés qui en beaucoup de points se rapprochent des monocristaux.

Bien que dans le cas général les propriétés cristallographiques des couches minces restent assez discutables, il existe déjà des techniques de traitement après évaporation qui permettent d'améliorer considérablement la stoechiométrie, et pour les cristaux uniaxes tels que le CdS l'orientation des cristallites par rapport au substrat. Ces mêmes traitements permettent aussi un meilleur contrôle des propriétés électriques c'est-à-dire des " dopages " beaucoup plus facilement réalisables que dans le cas des monocristaux.

Outre les nombreuses applications qui présentent d'incontestables intérêts économiques, ces techniques permettent l'obtention de matériaux qui facilitent grandement les études fondamentales.

La surface n'étant plus négligeable par rapport au volume, les propriétés de surface prennent dans ce cas une importance particulière. L'étude systématique des propriétés de surface est susceptible de fournir des données nécessaires à la connaissance la plus complète des couches minces.

En particulier, les constantes optiques permettant la détermination des transitions électroniques dues à l'action de la lumière peuvent être déduites de mesures de réflectivité pour lesquelles les couches minces sont les mieux adaptées.

Les phénomènes de transport dépendent essentiellement des états de surface et sont le mieux étudiés par les méthodes de la physique des surfaces.

Les effets photoélectriques, aussi bien la photoconductivité que l'effet photovoltaïque ont été étudiés par le passé essentiellement dans le monocristal. Ce n'est que récemment qu'une étude complète de la photoconductivité des couches minces de CdS [1] a été publiée. Nous reprendrons dans un premier chapitre les résultats essentiels de cette étude.

Bien que des réalisations importantes dans le domaine des piles solaires utilisant des couches minces aient déjà vu le jour, il ne semble pas qu'il y ait actuellement une théorie complète de l'effet photovoltaïque dans les couches minces de CdS par exemple. Une revue théorique de diverses possibilités d'interprétations de l'effet photovoltaïque dans les monocristaux photoconducteurs [2] a été donnée récemment sans pouvoir s'appuyer sur des résultats expérimentaux suffisants pour pouvoir confirmer sans ambiguité les prévisions théoriques.

Dans le présent travail sera exposée au chapitre 2 la base théorique de l'interprétation de l'effet photovoltaïque dans les couches minces de CdS en tenant compte essentiellement des caractéristiques d'interface $\mathrm{CdS}-\mathrm{Cu}_{2} \mathrm{~S}$. Dans le chapitre 3 seront donnés des résultats expérimentaux sur des photopiles en couches minces de CdS. Ces résultats tendent tous à indiquer que pour le CdS au moins, il s'agit bien d'une hétérojonction.

Une théorie tenant compte des propriétés spéci- 
fiques des couches minces et de l'interface rendrait assez bien compte de l'effet photovoltaïque.

2. Photoconductivité des couches minces de CdS. - Étant donné que les mêmes couches minces photosensibles de CdS sont utilisées comme photorésistances, photopiles et éléments actifs dans les transistors à couches minces, il semble intéressant d'analyser le phénomène de photoconduction en fonction des impuretés présentes dans le matériau.

La théorie de la photoconductivité des couches minces de $\operatorname{CdS}$ [1] est basée sur une généralisation de la statistique de Shockley et Read [3] et de Shockley [4] à l'étude de la variation de l'intensité du photocourant en fonction de la puissance du rayonnement incident. Les résultats montrent qu'il est possible d'interpréter la superlinéarité et la sublinéarité dans un photoconducteur par la présence de deux centres de recombinaison indépendants ou de trois niveaux dépendants.

L'effet photovoltaïque comme le photocourant dépendent de la densité des porteurs créés par la lumière et subsistant dans un régime stationnaire en tant que porteurs libres. Il est donc nécessaire d'examiner la statistique de recombinaison des porteurs créés par la lumière afin de déterminer le rôle joué par les impuretés définies par leur section efficace de capture.

\subsection{Statistique de recombinaison.}

2.1.a. Deux centres de recombinaison indépendants. - Un photoconducteur éclairé par un rayonnement d'énergie $h \nu$ supérieure à la largeur de la bande interdite $\varepsilon_{\mathrm{G}} \mathrm{du}$ matériau reçoit un nombre $Z$ de paires électron-trou produites par unité de temps :

$$
Z=u_{1}+u_{2}
$$

$u_{1}$ étant le nombre d'électrons qui se recombinent par unité de temps à travers le centre de recombinaison $\mathrm{M}_{1}$ à la profondeur $E_{1}$, donné par

$$
u_{1}=\frac{\left(p n-n_{i}^{2}\right) C_{\mathrm{n} 1} C_{11}}{C_{\mathrm{n} 1}\left(n+n_{1}\right)+C_{\mathrm{p} 1}\left(p+p_{1}\right)},
$$

$n$ et $p$ étant le nombre d'électrons et de trous libres par unité de volume ;

$u_{2}$ a la même définition pour le centre $M_{2}$.

La condition de neutralité pour le semiconducteur de type $n$, quand $\Delta n \gg \Delta p$, s'écrit :

$$
n-n_{0}=N_{1} \Delta f_{1}+N_{2} \Delta f_{2},
$$

où $N_{1}$ est le nombre de centres $\mathrm{M}_{1}$ par unité de volume, $N_{2}$ le nombre de centres $\mathrm{M}_{2}, \Delta f_{1}$ et $\Delta f_{2}$ les variations des fonctions $f_{1}$ et $f_{2}$ données par l'équation (4.2) de la référence [3] correspondant aux variations $\Delta n=n-n_{0}$ et $\Delta p=p-p_{0}$ d'électrons et de trous libres. L'équation (2.3) est une équation du second degré en $p$ qui permet de déterminer $p$ en fonction de $n$ et des paramètres qui caractérisent les niveaux d'impuretés. En remplaçant $p$ dans l'équation explicite déduite de l'équation (2.1), on obtient une relation entre $n$ et $Z$.

2.1.b. Trois niveaux dépendants. - Dans le cas où on considère que les impuretés peuvent être excitées dans les états de charge $s e,(s+1) e$ et $(s+2) e$, $s$ entier, le nombre $Z$ de paires électron-trou produites par unité de temps en régime stationnaire est

$$
Z=U\left(s+\frac{1}{2}\right)+U\left(s+\frac{3}{2}\right) .
$$

$U\left(s+\frac{1}{2}\right)$ étant le nombre de paires électron trou qui se recombinent en régime stationnaire en effectuant des transitions $s \rightarrow(s+1)$ par unité de temps :

$$
U\left(s+\frac{1}{2}\right)=N_{s}+\left(N_{s+1}\right) \frac{p n-n_{\mathbf{i}}^{2}}{\frac{1}{C(n, s)}\left[p+p^{*}\left(s+\frac{1}{2}\right)\right]+\frac{1}{C(p, S+1)}\left[n+n^{*}\left(s+\frac{1}{2}\right)\right]}
$$

$N_{\mathrm{s}}$ : nombre de niveaux par unité de volume dans l'état de charge $s e$;

$C(n, s):$ probabilité moyenne de capture d'un électron par unité de temps à travers un niveau dans l'état de charge se ;

$C(p, s)$ : probabilité moyenne de capture d'un trou par unité de temps à travers un niveau dans l'état de charge $s e$;

$$
\begin{aligned}
& n^{*}\left(s+\frac{1}{2}\right)=\frac{e(p, s)}{C(n, s)} \\
& p^{*}\left(s+\frac{1}{2}\right)=\frac{e(n, s+1)}{C(n, s+1)}
\end{aligned}
$$

$e(n, s)$ : probabilité moyenne d'émission d'un électron par unité de temps à travers un niveau dans l'état de charge se ;

$e(p, s)$ : probabilité moyenne d'émission d'un trou par unité de temps à travers un niveau dans l'état de charge $s e$.

La condition de neutralité nous donne à nouveau la relation entre $n$ et $Z$ qui, d'après les valeurs particulières des paramètres, conduit à une superlinéarité ou à une sublinéarité.

2.2. Sections efficaces de capture. - Dans les conditions physiques qui sont celles des couches minces de $\operatorname{CdS}$ [1], l'équation d'évolution d'excès 
de porteurs de charge libres avec le temps s'écrit :

$\frac{\mathrm{d}(\Delta n)}{\mathrm{d} t}=-\frac{C_{n}}{N_{1}} \Delta n\left[\frac{N_{1} n_{1}}{n_{0}}+n_{0}+\Delta n\right]$.

Pour une intensité de lumière incidente élevée même avec une grande concentration d'impuretés, lorsque le niveau d'impureté est situé bien audessous du niveau de Fermi, nous avons la condition :

$$
\Delta n \gg \frac{N_{1} n_{1}}{n_{0}}
$$

ce qui conduit ì :

$$
\frac{\mathrm{d}(\Delta n)}{\mathrm{d} t}=-\frac{C_{n}}{N_{1}} \Delta n^{2}=-v \sigma_{n} \Delta n^{2},
$$

où $v$ est la vitesse thermique des électrons et $\sigma_{n}$ est la valeur moyenne de la section efficace de capture d'un centre pour les électrons.

L'intégration de cette équation donne :

$$
\frac{1}{\Delta n}=v \sigma_{n} t+\frac{n^{\prime}}{1} \text {. }
$$

Avec $n^{\prime}$ constant, cette équation montre que nous avons à faire à un mécanisme bimoléculaire.

L'étude expérimentale de la décroissance de la photoconductivité est très riche de renseignements et donne des résultats dépendant aussi bien de la température, de l'jntensité d'éclairement que de la nature des défauts.

Pour interpréter l'ensemble des résultats, il semble nécessaire dadmettre au moins trois niveaux d'impuretés, chacun étant prédominant dans un intervalle de température et d'éclairage donné :

10 Un centre $M_{1}$ avec une section efficace de capture pour les électrons assez élevée, et tel que le remplissage de ce niveau soit très peu affecté par une élévation de température jusqu'à 500 oK. Un tel centre pourrait expliquer la décroissance très rapide de la photoconductivité à $500^{\circ} \mathrm{K}$.

20 Un centre $M_{2}$ avec une section efficace de capture pour les électrons inférieure à la section efficace des centres $M_{1}$ et tel que le remplissage de ce niveau soit considérablement affecté par une élévation de température à $500^{\circ} \mathrm{K}$.

$3^{\circ}$ Un ou plusieurs centres qui déterminent la décroissance très lente de la photoconductivité aux basses températures.

Nous admettons ainsi que les courbes de décroissance de photoconductivité obtenues avec des éclairages diff'́rents, mettent en évidence des centres de recombinaison différents dont l'importance relative change avec la température.

L'analyse des courbes de décroissance à $500^{\circ} \mathrm{K}$ montre que l"inverse du nombre de porteurs libres est proportionnel au temps (mécanisme bimoléculaire) (fig. 1, courbe $a$ ).

Si l'on admet que seuls les centres $M_{1}$ interviennent dans la décroissance de la photoconduc-

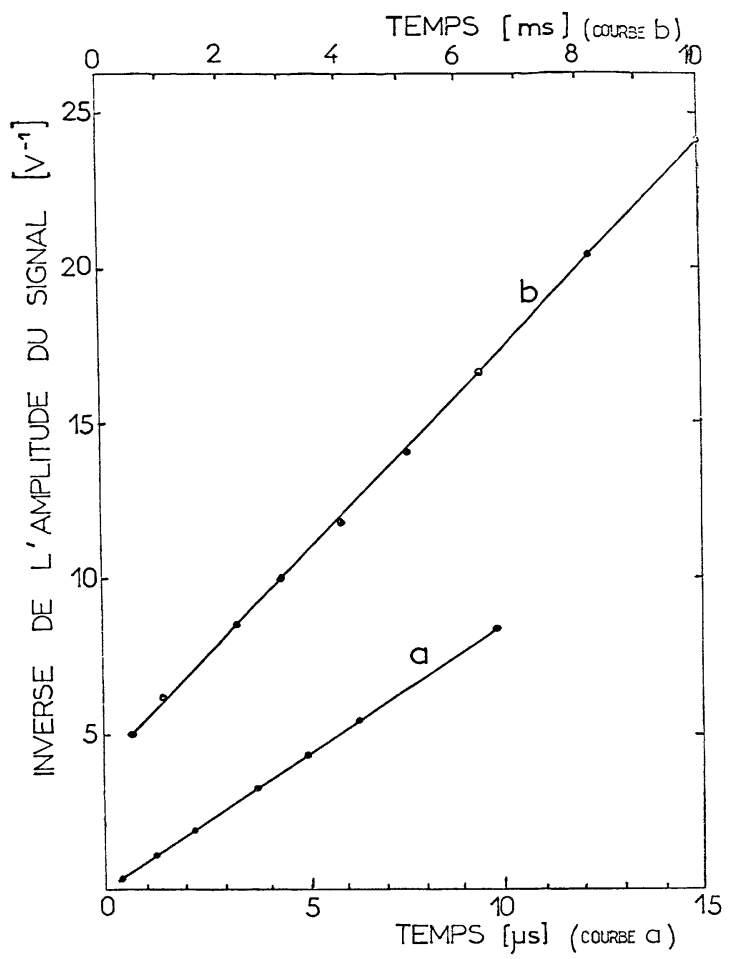

FIg. 1. - Variation de l'inverse de l'amplitude du signal dans les couches minces photorésistantes en fonction du temps.

Courbe $a: T=500{ }^{\circ} \mathrm{K}$; Courbe $b: T=77^{\circ} \mathrm{K}$ et 300 oK.

tivité. leur section efficace peut être calculée à partir de la pente de la droite obtenue.

A la température ambiante et à $77^{\circ} \mathrm{K}$, une partie des trous créés par la lumière va être captée par les centres $M_{1}$ et une autre partie par les centres $\mathbf{M}_{2}$. La proportion des trous captés par ces deux niveaux va dépendre fortement de la température, de l'intensité et de la durée de l'éclairage. Si la durée de l'éclairage atteint une certaine valeur, nous pouvons admettre que la partie finale des courbes de décroissance est due aux centres $\mathrm{M}_{2}$, et nous avons constaté aussi dans un certain nombre de cellules que l'inverse du nombre de porteurs libres est proportionnel au temps (mécanisme bimoléculaire) (fig. 1, courbe $b$ ), d'où la détermination de la section efficace de capture caractéristique de $\mathrm{M}_{2}$ à $77^{\circ} \mathrm{K}$. Il en est de même pour un certain nombre de cellules à 300 oK.

Ces expériences ont été réalisées sur différents types de cellules avec ou sans dopage au cuivre et à l'argent, les résultats sont semblables.

Dans toutes les couches de CdS on vérifie l'existence de deux niveaux auxquels nous associons les sections efficaces suivantes :

$$
\begin{aligned}
& E_{1} \simeq 0,52 \mathrm{eV}, \quad \sigma_{1}<10^{-17} \mathrm{~cm}^{2} \\
& E_{2} \simeq 1,2 \mathrm{eV}, \quad \sigma_{2} \simeq 10^{-19} \mathrm{~cm}^{2} .
\end{aligned}
$$


Nous avons trouvé aussi des niveaux moins profonds avec les énergies d'ionisation $0,34 \mathrm{eV}$ et $0,25 \mathrm{eV}$. Ces niveaux peuvent avoir un comportement de pièges ou centres de recombinaison avec des sections efficaces de capture très petites pour les électrons, dépendant de la position du niveau de Fermi et de la température.

Les métaux étudiés, comme par exemple l'argent et le cuivre, vont créer des défauts du réseau tels que les électrons associés à ces niveaux aient des énergies d'ionisation très élevés. Ces centres doivent avoir ainsi une charge positive par rapport au réseau et dans ces conditions les sections efficaces de capture pour les électrons doivent être élevées, de l'ordre de $10^{-15} \mathrm{~cm}^{2}$ [5]. La durée de vie moyenne des électrons qui se recombinent à travers ces centres devrait être petite, inférieure à celles que nous pouvions mesurer.

La section efficace de $10^{-17} \mathrm{~cm}^{2}$ correspond au niveau dont l'énergie d'ionisation est $1,2 \mathrm{eV}$. Cette valeur laisse supposer qu'il s'agit d'un centre neutre [5].

Le niveau $0,52 \mathrm{eV}$ avec une section efficace $<10^{-19} \mathrm{~cm}^{2}$ joue un rôle important dans la région de photoconductivité élevée. Ces centres doivent avoir une structure assez complexe et pourraient etre formés, soit d'atomes interstitiels de cadmium, soit de groupes de lacunes de soufre (une lacune de soufre est un niveau donneur avec une énergie d'ionisation $0,03 \mathrm{eV}[6])$.

L'étude expérimentale et théorique des défauts du réseau dans les couches minces de sulfure de cadmium montre qu'il est nécessaire d'avoir un excès de cadmium pour obtenir des couches minces photosensibles.

Cet excès de cadmium joue un rôle important dans la formation de centres de faibles sections efficaces de capture. Ces centres ont probablement une nature complexe.

C'est la variation du taux d'excès de cadmium qui détermine le degré de photosensibilité, car c'est de lui que dépend la formation de centres avec très faible section efficace de capture, et par conséquent de grande durée de vie des électrons.

Un trop grand excès de cadmium conduit à un phénomène de saturation. Les couches ainsi obtenues sont peu photosensibles et accusent une fatigue trop rapide en fonction de la durée d'illumination.

\section{Effet photovoltaïque de $\mathbf{C d S}$ en couches minces.} - La théorie de l'effet photovoltaïque dans le cas des piles solaires en couches minces peut être élaborée en prenant en considération le fait que les dimensions mêmes des couches imposent des conditions aux limites. Une couche peut être considérée infinie dans les deux directions dans le plan, mais son épaisseur est telle qu'elle détermine la distribution de charges électriques tout à travers de la couche, étant donnée l'existence des états de surface de part et d'autre. Pour une couche suffisamment épaisse et contenant un nombre de porteurs de charge élevé, il sera possible de considérer qu'elle n'a les propriétés intrinsèques du semiconducteur qu'à une profondeur assez grande. Si l'on admet une densité de charge en surface de $10^{15} \mathrm{~cm}^{-2}$, elle ne serait neutralisée dans un semiconducteur à $10^{19}$ électrons $\mathrm{cm}^{-3}$ qu'à une distance de $10^{-4} \mathrm{~cm}$ de la surface, c'est-à-dire à un micron de profondeur.

Doper une couche en surface afin d'obtenir une jonction à effet photovoltaïque équivaut à construire une deuxième couche à caractéristiques différentes. La jonction est dans ce cas une hétérojonction formée à l'interface des deux couches. Le potentiel d'une telle jonction est donné par la distribution de charges électriques de part et d'autre de l'interface. L'action de la lumière modifie la distribution des charges au niveau de l'interface et détermine la différence de potentiel et le courant dans la photopile.

Considérons successivement les surfaces de deux couches $n$ et $p$ afin d'examiner leur superposition pour former la jonction.

\subsection{Charge d'espace et barrière de potentiel} A LA SURface d'une COUGhe de tyPe n. - Considérons la surface d'une couche de type $n$. La charge d'espace est constituée par les donneurs ionisés. Le niveau de Fermi $\varepsilon_{F}$ à l'équilibre thermodynamique arrive perpendiculairement à la surface, mais les bandes sont incurvées vers le haut en raison de la présence de centres ionisés localisés en surface ou d'un potentiel extérieur.

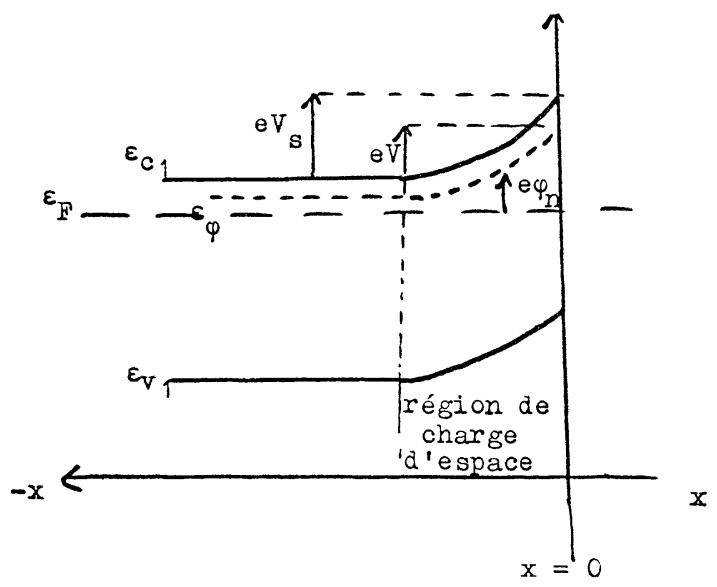

Fig. 2. - Schéma d'énergie d'une surface de semiconducteur de type $n$ avec une région d'appauvrissement.

La largeur de la bande interdite à l'intérieur du semiconducteur est $\varepsilon_{\mathrm{G}_{1}}=\varepsilon_{\mathrm{c}_{1}}-\varepsilon_{\mathrm{v}_{\mathbf{1}}}$. La barrière de potentiel mesurće au-dessus de la bande de conduction est $V$, le potentiel de surface est $V_{\mathrm{B}}$. La densité 
de porteurs en terme de densité intrinsèque $n_{\mathrm{i}}$ est donnée par :

$n=n_{\mathrm{i}} \exp \left[\frac{e\left(V-\varphi_{n}\right)}{k T}\right]=n_{\mathrm{b}} \exp (e V / k T)$,

$n_{\mathrm{b}}$ étant la densité de porteurs libres dans la masse de semiconducteur; $\varphi$ est la position du quasiniveau de Fermi seulement dans des conditions hors d'équilibre en raison d'une barrière de potentiel près de la surface ou à cause de l'action de la lumière.

La forme du potentiel électrostatique à l'approche de la surface est donnée par des solutions de l'équation de Poisson :

$$
\frac{\mathrm{d}^{2} V}{\mathrm{~d} x^{2}}=-\frac{\rho}{x_{1} \varepsilon_{0}}
$$

$x_{1}=$ constante diélectrique du semiconducteur,

$\varepsilon_{0}=$ perméabilité du vide.

La densité de charge est :

$$
\begin{aligned}
\rho(-x)=e[(p & \left.\left.-p_{b}\right)-\left(n-n_{b}\right)\right] \\
& =e\left[n_{b_{1}}-n_{b_{1}} \exp (e V / k T)\right. \\
& \left.+p_{b_{1}} \exp -e V(k T)-p_{b_{1}}\right] .
\end{aligned}
$$

Done :

$$
\begin{aligned}
\frac{\mathrm{d}^{2} V}{\mathrm{~d} x^{2}}=\frac{e}{\varkappa_{1} \varepsilon_{0}}\left[p_{b_{1}}-p_{b_{1}} \exp (-e V / k T)\right. \\
\left.-n_{b_{1}}+n_{b_{1}} \exp (e V / k T)\right] .
\end{aligned}
$$

3.2. Charge D'espace et barrière de potentiel A LA SURFACE D'UNe COUChe DE TYPE $p$. - A la surface d'une couche de type $p$, la charge d'espace est constituée par des accepteurs ionisés. La largeur de la bande interdite est : $\varepsilon_{\mathrm{G}_{2}}=\varepsilon_{\mathrm{c}_{2}}-\varepsilon_{\mathrm{v}_{\mathrm{i}}}$. Les définitions de la barrière de potentiel par rapport à la bande de valence $V$ et du quasi-niveau de Fermi $e \varphi$ par rapport au niveau de Fermi sont analogues à ceux pour le semiconducteur de type $n$. La densité de porteurs est maintenant donnée par :

$p=p_{i} \exp \left[-\frac{e\left(V-\varphi_{p}\right)}{k T}\right]=p_{b} \exp (-e V / k T)$.

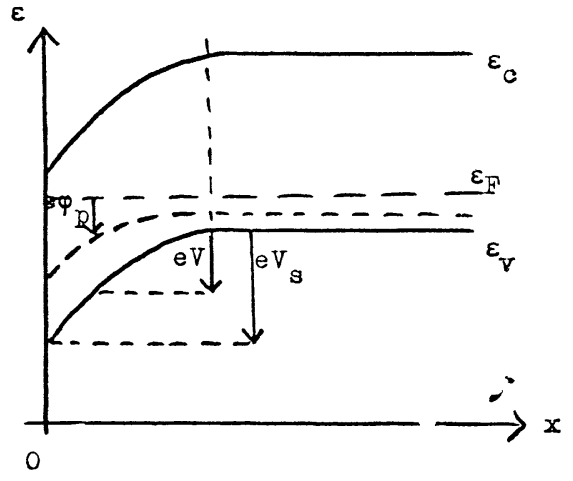

Fıg. 3. - Schéma d'énergie d'une surface de semiconducteur de type $p$ avec une région d'appauvrissement.
La forme du poteritiel électrostatique à l'approche de la surface sera de nouveau donnée par l'équation de Poisson :

$$
\begin{aligned}
\frac{\mathrm{d}^{2} V}{\mathrm{~d} x^{2}}=\frac{e}{x_{2} \varepsilon_{0}} & {\left[\left(p_{b_{\mathrm{i}}} \exp (-e V / k T)\right.\right.} \\
& \left.-p_{b_{\mathbf{s}}}+n_{b_{2}}-n_{b_{2}} \exp (e V / k T)\right] .
\end{aligned}
$$

3.3. Jonction A L'interface de Deux Couches DE SEMICONDUCTEURS DIFFÉRENTS. - Considérons la première couche de semiconducteur de type $n$, de bande interdite $\varepsilon_{G_{1}}$, à laquelle vient se superposer une couche de type $p$ avec une bande interdite $\varepsilon_{\mathbf{G}_{2}}$. L'interface des deux couches formera une jonction $n-p$ au voisinage de laquelle la structure de bande sera fortement déformée.

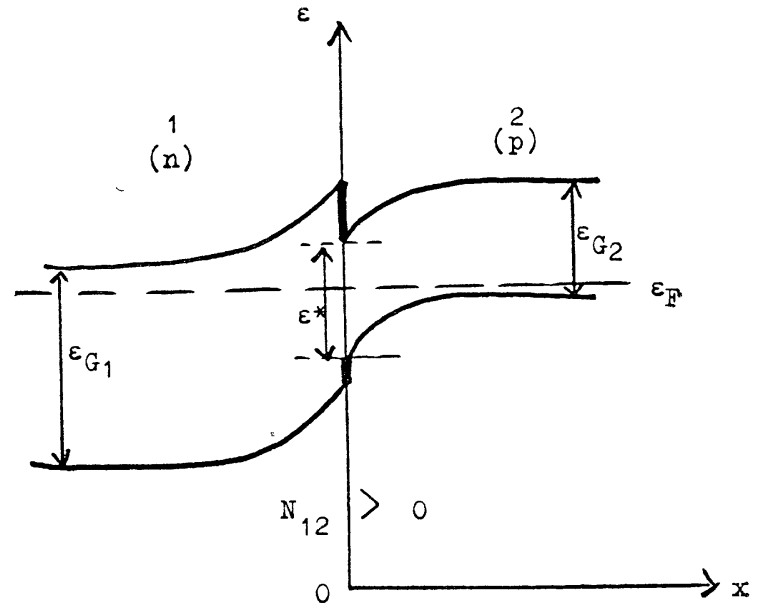

Ftg. 4. - Représentation schématique du diagramme d'énergie d'une hétérojonction à l'interface de deux couches de semiconducteurs différents.

Au voisinage de la jonction, la différence de potentiel électrostatique définit une région de charge d'espace dans laquelle la notion de structure de bande reste encore valable avee les définitions données précédemment.

A l'interface même des deux semiconducteurs, la structure de bande n'a plus de signification et la structure électronique est donnée par un ensemble d'états localisés. Les états à l'interface étant ionisés donnent lieu à une densité de charge dans le plan de la jonction, alors qu'une telle charge n'existe pas dans l'homojonction. Appelons $N_{12}>0$ la densité de charge totale à l'interface. $N_{12}$ est définie par la condition de neutralité électrique de l'ensemble

$$
\begin{aligned}
N_{12}=\int_{-\infty}^{0} & {\left[N_{1}-n_{1}(x)+p_{1}(x)\right] \mathrm{d} x } \\
& -\int^{\infty}\left[P_{2}+n_{2}(x)-p_{2}(x)\right] \mathrm{d} x,
\end{aligned}
$$

$N_{1}$ et $P_{2}$ sont respectivement les densités fixes de 
donneurs dans le semiconducteur 1 et d'accepteurs dans le semiconducteur 2 .

Avec un potentiel réduit $v_{1}=e V_{1} / k T$ pour le premier semiconducteur et $v_{2}=e V_{2} / k T$ pour le second semiconducteur, la distribution du potentiel de part et d'autre de l'interface sera :

- à gauche de la jonction :

$$
\begin{array}{r}
\frac{\mathrm{d}^{2} v_{1}}{\mathrm{~d} x^{2}}=-\frac{e^{2}}{x_{1} \varepsilon_{0} k T}\left[n_{b_{1}}-p_{b_{1}}-n_{b_{1}}\right. \\
\left.\exp \left(v_{1}\right)+p_{b_{1}} \exp \left(-v_{1}\right)\right],
\end{array}
$$

— et à droite :

$$
\begin{array}{r}
\frac{\mathrm{d}^{2} v_{2}}{\mathrm{~d} x^{2}}=-\frac{e^{2}}{\chi_{2} \varepsilon_{0} k T}\left[p_{b_{2}}-n_{b_{2}}-p_{b_{2}} \exp \left(-v_{2}\right)\right. \\
\left.+n_{b_{2}} \exp \left(v_{2}\right)\right], \quad
\end{array}
$$

avec les conditions aux limites :

$$
\begin{array}{lll}
v_{1} \rightarrow 0 & \text { quand } & x \rightarrow-\infty \\
v_{2} \rightarrow 0 & \text { quand } & x \rightarrow \infty
\end{array}
$$

Loin de l'interface, les positions des deux bandes de conduction, respectivement $\varepsilon_{c_{1}}, \varepsilon_{c_{1}}$, ainsi que celles des deux bandes de valence $\varepsilon_{v_{1}}, \varepsilon_{v}$, sont connues, et le niveau de Fermi est constant à travers l'ensemble.

3.4. Régime Quasi-Stationnaire. - Lorsqu'une cellule solaire constituée par une hétérojonction est soumise à l'action de la lumière, une densité de porteurs de charges positive et négative proportionnelle au flux est créée au niveau de l'interfáce. L'énergie nécessaire à la création d'une paire électron-trou est $\varepsilon^{*}$, l'énergie de la bande interdite effective correspondant à la région d'états localisés. Cette énergie est nécessairement inférieure à la bande interdite des deux semiconducteurs formant l'hétérojonction. Elle est au maximum égale à la largeur de la bande interdite la plus faible. Dans le cas des piles solaires au CdS, la réponse spectrale est donnée sur la figure 5 .

Le maximum de réponse de la cellule est aux environs de $6500 \AA$, alors que la bande interdite de CdS correspond à $5100 \AA$.

Sur la figure 6 est donnée la réponse spectrale pour la même photopile à trois intensités d'irradiation différentes. On constate que, à mesure que la densité d'énergie incidente augmente, la réponse spectrale se déplace vers les faibles énergies, c'est-àdire $\varsigma^{*}$ diminue. A faible intensité, seuls les centres de faible section efficace sont ionisés. A mesure que la densité d'énergie incidente augmente, les niveaux de section efficace de capture plus élevée, et donc de plus faible énergie, sont aussi ionisés ; ce qui est en accord aussi avec les valeurs des sections efficaces de capture déduites des mesures de photoconductivité. On peut penser que les niveaux localisés forment une bande d'énergie presque continue.

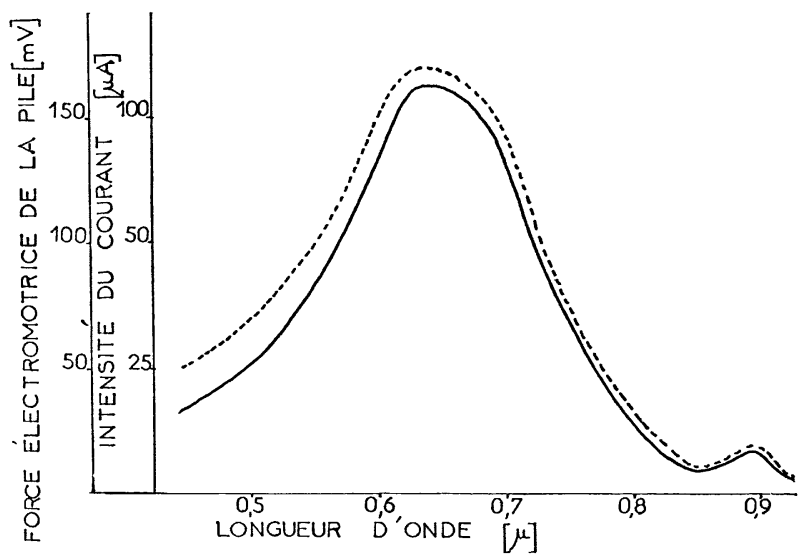

FIG. 5. - Réponse spectrale à énergie constante, $2 \mathrm{~mW} / \mathrm{cm}^{2}$, pour une cellule photovoltaïque de CdS de surface $1 \mathrm{~cm}^{2}$.

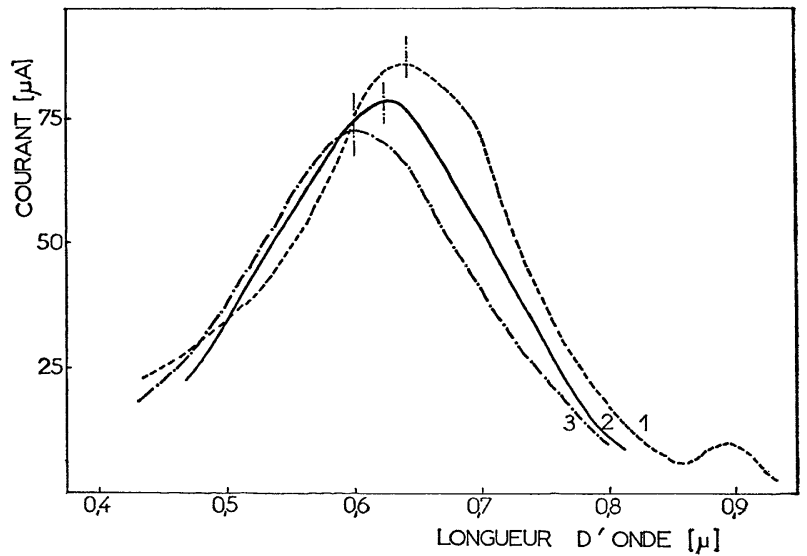

Fig. 6. - Réponses spectrales d'une photopile de CdS en couche mince correspondant à différentes intensités d'irradiation :

Courbe $1: 2,4 \mathrm{~mW} / \mathrm{cm}^{2}$;

Courbe $2: 2,6 \mathrm{~mW} / \mathrm{cm}^{2}$;

Courbe $3: 1,2 \mathrm{~mW} / \mathrm{cm}^{2}$.

L'épaisseur de l'interface étant extrêmement faible, de quelques distances interatomiques, la largeur de la région de niveaux localisés est très réduite, et par conséquent la zone de création de paires électron-trou est très restreinte, inférieure à la longueur de diffusion $\sqrt{\bar{D} \tau}$ de porteurs libres de part et d'autre de l'interface. Une telle situation facilite grandement le calcul, étant donné que l'on peut négliger la recombinaison.

Dans le cas où on s'intéresse uniquement à la phototension et sans qu'aucun champ extérieur soit appliqué, le schéma énergétique est analogue à celui du problème à l'équilibre, mais maintenant avec des conditions aux limites différentes. Loin de l'interface, la position des niveaux de Fermi et la densité de porteurs sont données par les conditions d'équilibre, et correspond à ceux du semiconducteur formant la couche de chaque côté de l'interface. 


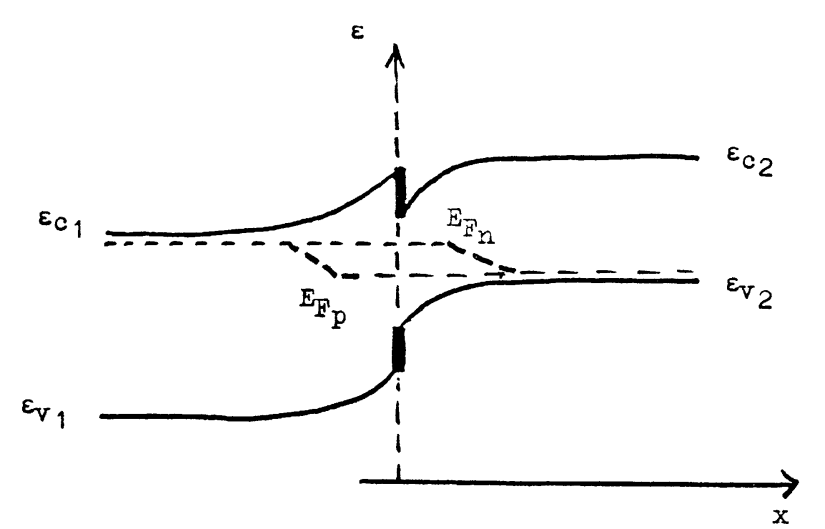

FIg. 7. - Schéma des bandes d'énergie au voisinage d'une hétérojonction et des quasi-niveaux de Fermi en régime hors d'équilibre.

A l'interface on doit considérer les quasi-niveaux de Fermi $E_{F_{n}}$ et $E_{F_{p}}$.

La densité des porteurs dans la région de charge d'espace est donnée en fonction des quasi-niveaux de Fermi :

$$
\begin{aligned}
& n_{b}^{*}=N_{c} \exp \left[\frac{\varepsilon_{c}-\varepsilon_{F_{n}}}{k T}\right], \\
& p_{b}^{*}=N_{v} \exp \left[-\frac{\varepsilon_{v}-\varepsilon_{t p}}{k T}\right] .
\end{aligned}
$$

Pour un semiconducteur de large bande interdite comportant une grande densité de donneurs, le quasi-niveau des porteurs majoritaires doit pratiquement coïncider avec le niveau de Fermi du semiconducteur- $n$, alors que le quasi-niveau des porteurs minoritaires peut être largement différent.

La création par l'illumination de paires électrons $\left(\delta n_{b}\right)$ - trous $\left(\delta p_{b}\right)$ conduit à

$$
n_{b}^{*}=n_{b}+\delta n_{b} \simeq n_{b}
$$

alors que

$$
p_{b}^{*}=p_{b}+\delta p_{h}-\delta p_{b}
$$

L'équation de neutralité dans la région efficace est :

$$
\begin{aligned}
N_{12}^{*}=\int_{-\infty}^{0} & {\left[N_{1}-n_{1}^{*}(x)+p_{1}^{*}(x)\right] \mathrm{d} x } \\
& -\int_{0}^{\infty}\left[P_{2}+n_{2}(x)-p_{2}(x)\right] \mathrm{d} x .
\end{aligned}
$$

Les équations de Poisson correspondantes donnent maintenant la distribution de charge dans la région efficace, donc des quantités étoiles, ceci bien entendu à condition de pouvoir considérer les quasiniveaux de Fermi constants à travers la région efficace sous l'action de la lumière.

Les quasi-niveaux de Fermi ne sont pas constants dans le cas général à travers la jonction, et il est alors nécessaire de considérer la diffusion des porteurs en tenant compte du champ électrique de la région d'espace. La condition d'équilibre permet d'écrire qu'en tout point d'abscisse $x$ le courant est nul.

Écrivons le courant d'électrons et de trous à gauche de l'interface :

$$
\begin{gathered}
J_{n}(x)=e \ell_{n_{1}} n_{1}^{*}(x) E_{1}(x)+e D_{n_{1}}\left(\mathrm{~d} n_{1}^{*} / \mathrm{d} x\right), \\
J_{p_{1}}(x)=e \mu_{p_{1}} p_{1}^{*}(x) E_{1}(x)-e D_{p_{1}}\left(\mathrm{~d} p_{1}^{*} / \mathrm{d} x\right), \\
J_{1}=J_{n_{1}}+J_{p_{1}}
\end{gathered}
$$

et à droite de l'interface :

$$
\begin{gathered}
J_{n_{\mathrm{z}}}(x)=e \mu_{n_{2}} n_{2}^{*}(x) E_{2}(x)+e D_{n_{2}}\left(\mathrm{~d} n_{2}^{*} / \mathrm{d} x\right) \\
J_{p_{2}}(x)=e u_{p_{2}} p_{2}^{*}(x) E_{2}(x)-e D_{p_{\mathbf{z}}}\left(\mathrm{d} p_{2}^{*} / \mathrm{d} x\right) \\
J_{2}=J_{p_{2}}+J_{n_{2}}
\end{gathered}
$$

à une dimension.

A la jonction, nous avons la condition

$$
J_{1}(0)=J_{1}(0) \text {. }
$$

L'intégration des équations de Poisson,

$$
\mathrm{d}^{2} V / \mathrm{d} x^{2}=-\rho / x \varepsilon_{6},
$$

de part et d'autre de la jonction donne :

$$
E_{1}=-\mathrm{d} V_{1} / \mathrm{d} x \text { et } E_{2}=-\mathrm{d} V_{2} / \mathrm{d} x,
$$

et en utilisant la statistique de Maxwell-Boltzmann, on déduit :

$$
n^{*}=A \exp (e V / k T) \text { et } p^{*}=B \exp (-e V / k T) \text {. }
$$

11 est possible maintenant d'entreprendre des calculs numériques dans le cas précis afin de déduire des ordres de graudeur pour les quantités à comparer avec l'expérience.

Il est évident que pour diverses situations expérimentales, il est possible d'apporter des détails supplémentaires sur la statistique de recombinaison, sur les niveaux localisés à l'interface et de déduire avec exactitude l'allure des bandes d'énergie dans la région de charge d'espace.

Comme dans de nombreux cas d'étude théorique de l'effet photovoltaïque au niveau d'une jonction $n-p$, ici aussi le rôle des porteurs de charge minoritaires est essentiel pour l'établissement de la phototension. Les paires électron-trou créées au niveau de l'interface seront séparées par l'effet des régions d'appauvrissement des deux côtés de la jonction. Les trous créés optiquement diffusent vers l'interface et sont accélérés à travers la région d'appauvrissement par le champ électrostatique existant à l'interface.

Une étude de la statistique de recombinaison des trous a été publiée récemment [7] dans le cas de piles solaires de CdS recouvertes d'une couche de cuivre. 
4. Etude expérimentale des photopiles au CdS. 4.1. Réalisation de piles Solaires en couches MINCES DE CdS. - Nos recherches ont permis de montrer qu'il était possible de fabriquer des cellules photovoltaïques ayant pour élément actif une couche mince de CdS évaporée sous vide dont le rendement énergétique atteint environ 1,5\%.

Les divers substrats utilisés sont : le verre métallisé (aluminium), la feuille d'aluminium mince $(50 \mu)$, le film plastique métallisé (Kapton de Dupont de Nemours en épaisseur 25 ou $50 \mu$ doré ou aluminé sous vide).

Notre orientation vers les deux derniers a été motivée par les caractéristiques intéressantes qu'ils confèrent aux photopiles dont ils constituent la base : légèreté, mise en forme possible sur des rayons de courbure faible, possibilités de réalisation de panneaux intégrés.

Quel que soit le substrat utilisé, la technique de fabrication est la même et les opérations effectuées sont les suivantes :

Nettoyage des substrats, évaporation de CdS environ $20 \mu$, évaporation d'une couche semi-transparente de cuivre, évaporation de grilles métalliques : cuivre, or, etc..., recuisson aux environs de $300{ }^{\circ} \mathrm{C}$, applications de solutions contenant des ions $\mathrm{Cl}-$, recuisson de stabilisation, décapage superficiel par attaque chimique, protection par vernis polymérisable.

Nous nous sommes attachés à rendre l'ensemble de ces opérations reproductibles d'une évaporation à l'autre par la mesure systématique du plus grand nombre de paramètres possible et leur contrôle, ce qui nous permet d'avoir une dispersion des résultats inférieure à $10 \%$ sur deux évaporations effectuées dans les mêmes conditions.

L'évaporation flash a dû être abandonnée en raison du chauffage trop important des cibles provoqué par le ruban incandescent et des pertes excessives de CdS qu'elle provoquait ; le CdS est donc évaporé classiquement dans un creuset de tantale chauffé directement par effet Joule. La vitesse d'évaporation est réglée par action sur l'intensité qui le traverse, tandis que l'épaisseur du dépôt est mesurée par un dispositif optique utilisant les interférences produites par une couche de référence (dépôt sur verre optique).

Le chauffage des cibles est effectué par rayonnement d'un ruban chauffé par effet Joule, tandis que leur température est mesurée par des thermocouples solidaires du plateau tournant ; la constance de l'épaisseur est assurée par la rotation de ce dernier.

Pendant l'évaporation, le vide est maintenu aux environs de quelques $10^{-6} \mathrm{~mm}$ de $\mathrm{Hg}$.

Après refroidissement, les couches obtenues sont prêtes pour le dépôt de la couche semi-transparente de cuivre; ce dépôt est effectué dans un banc de métallisation sous vide et son épaisseur est réglée avec précision par un áispositif à quartz (environ 1500 à $2000 \AA$ ).

Les grilles de collection du courant (cuivre ou or) sont ensuite évaporées dans le même banc.

A chaque évaporation un certain nombre de couches témoins sont réalisées sur plaquette de pyrex à faces polies optique, ce qui permet d'effectuer des mesures sur la couche de CdS elle-même.

Une étude cristallographique est faite par la méthode de Debye-Scherrer ; les couches sont polycristallines, mais il existe pour les cristaux formés une orientation préférentielle; l'axe optique [002] est approximativement perpendiculaire au support, l'écart angulaire maximum atteignant environ $40^{\circ}$.

Une étude des propriétés électriques est également effectuée ; l'épaisseur ayant été vérifiée par l'inscription d'interférences au spectromètre, des mesures de résistance et d'effet Hall sont faites, qui fournissent pour des couches conduisant à la réalisation de photopiles ayant un bon rendement énergétique, les caractéristiques suivantes :

Résistivité : $10^{-2}$ à $10^{-1} \Omega \times \mathrm{cm}$; mobilité : 6 à $15 \mathrm{~cm}^{2} \mathrm{~V}^{-1} \mathrm{~s}^{-1}$; nombre de porteurs : $10^{18}$ à $10^{20} \mathrm{~cm}^{-3}$.

\section{2. Étude eXPérimentale de L'effet photo-} voltaique DaNS LE CdS.

4.2.a. Caractéristiques d'utilisation $V=f(I)$. A énergie incidente constante. on trace le graphe représentant la tension utilisable en fonction du courant délité ; cette courbe est caractérisée par trois points principaux :

- J'intersection avec l'axe des $V$ représente la force électromotrice de la pile ou tension en circuit ouvert $V_{\text {co }}$;

- l'intersection avec l'axe des $I$ représente l'intensité fournie par la pile mise en court-circuit extérieur : $I_{\mathrm{cc}}$;

- le point de rendement maximum défini par le maximum du produit $V I$ (point de contact avec une hyperbole $V I=$ constante).

Le rendement énergétique est calculé pour les valeurs correspondantes de $V$ et de $I$.

La figure 8 est le graphe typique relevé pour une énergie incidente de $140 \mathrm{~mW} / \mathrm{cm}^{2}$ réalisant avec une bonne approximation une simulation solaire.

4.2.b. Réponse spectrale à énergie constante. - On trace les variations de $V_{\text {co }}$ et $I_{\text {cc }}$ en fonction de la longueur d'onde de la lumière incidente, le flux d'énergie étant maintenu constant. La courbe représentant la réponse spectrale est donnée sur la figure 5 comme il a déjà été indiqué.

Le maximum de l'effet photovoltaïque se situe à une longueur d'onde de $0,6 \mu$, supérieure à celle qui correspond au gap du CdS $(0,51 \mu)$.

4.2.c. Réponse spectrale à énergie variable. - Les courbes données sur la figure 6 sont tracées pour diverses valeurs de l'énergie incidente.

Il est à noter que le maximum se déplace vers les 


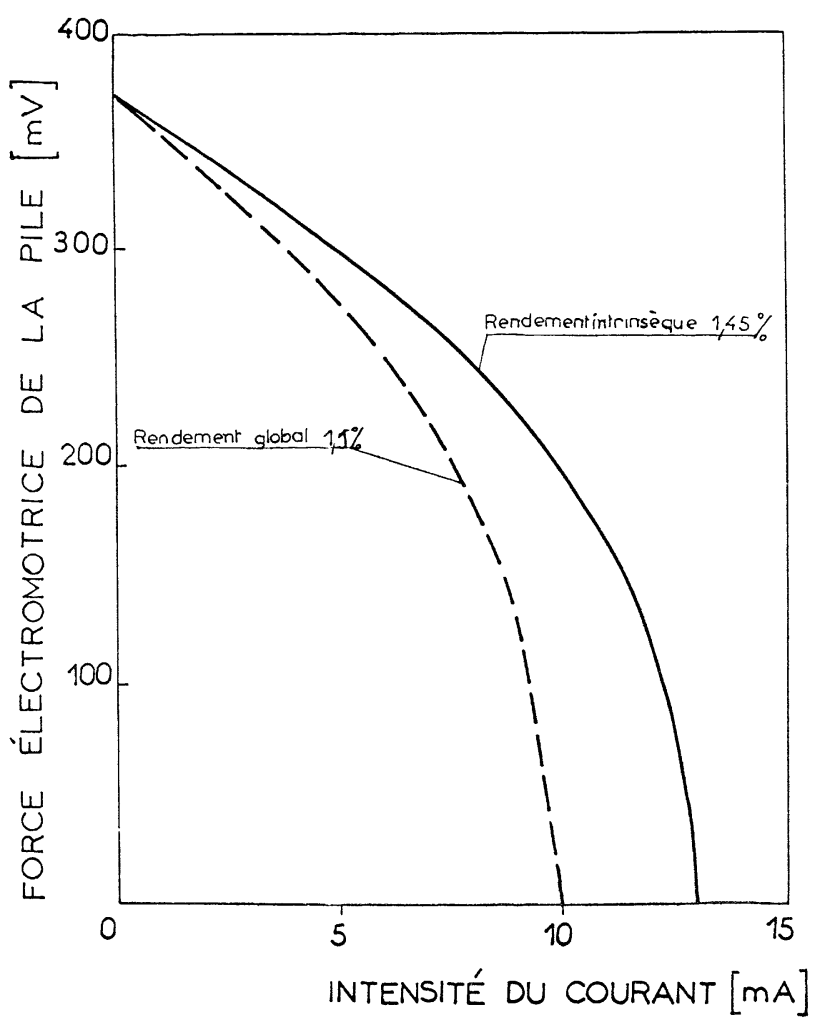

FIg. 8. - Caractéristique électrique $V=f(I)$ d'une photopile typique de CdS en couche mince.

laibles longueurs d'onde, c'est-à-dire vers la longueur d'onde correspondant au gap lorsque l'énergie décroît.

4.2.d. Rendement en fonction de l'énergie incidente. - $V_{\text {co }}$ et $I_{\text {cc }}$ sont mesurés pour diverses valeurs de

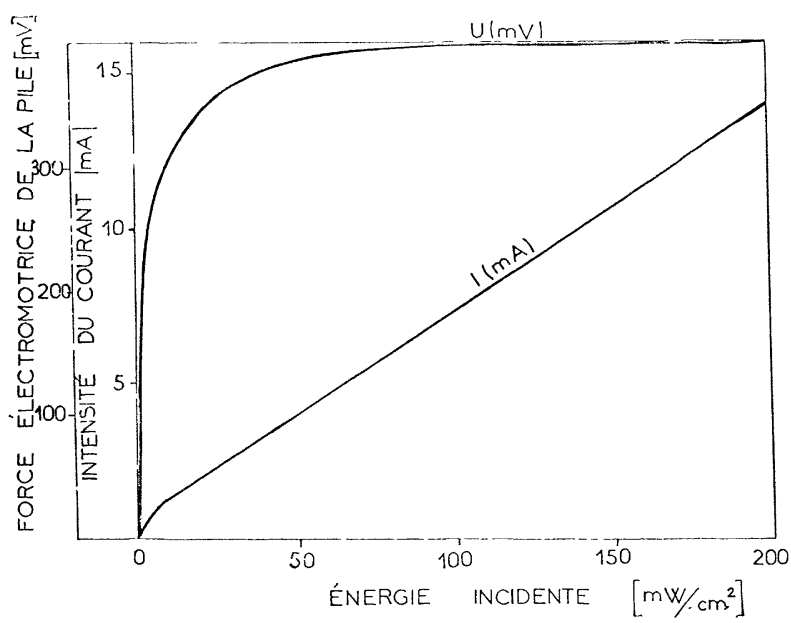

Fig. 9. - Force électromotrice de la pile de CdS, $U(\mathrm{mV})$, en fonction de l'intensité d'éclairement incident : Courbe $a$.

Courant de court-circuit $I_{\mathrm{cc}}(\mathrm{mA})$ en fonction de l'intensité d'éclairement incident : Courbe $b$. l'énergie incidente. La courbe représentative est donnée sur la figure 9 .

La force électromotrice est soumise à un phénomène de saturation dès $50 \mathrm{~mW} / \mathrm{cm}^{2}$ tandis que l'intensité en court-circuit est presque proportionnelle à l'éclairement.

4.2.e. Rendement en fonction de la température. Les variations de $V_{\text {co }}$ et $I_{\text {cc }}$ en fonction de la température dans le domaine $-80^{\circ} \mathrm{C}$ et $+60^{\circ} \mathrm{C}$ sont représentées sur la figure 10 . La forme de la courbe $V=f(I)$ restant la même, le rendement s'accroît lorsque la température diminue.

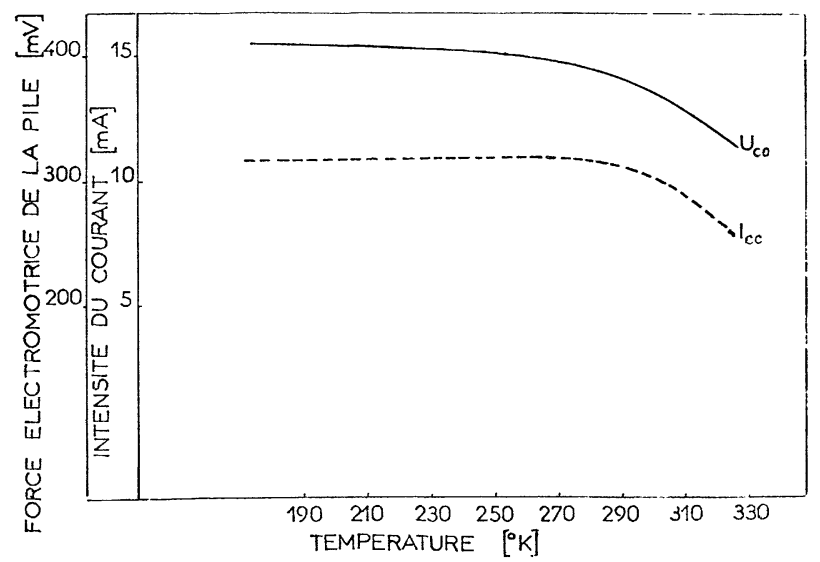

FIG. 10. - Caractéristiques $U_{\text {co }}$ et $I_{c}$ d'une photopile au CdS en fonction de la température.

4.2.f. Phénomènes de vieillissement. - Le rendement énergétique est mesuré à intervalles réguliers pendant une période de plusieurs mois, les cellules étant conservées à l'air libre. Les variations moyennes observées sont indiquées sur la figure 11. On remarque qu'une augmentation est bientôt

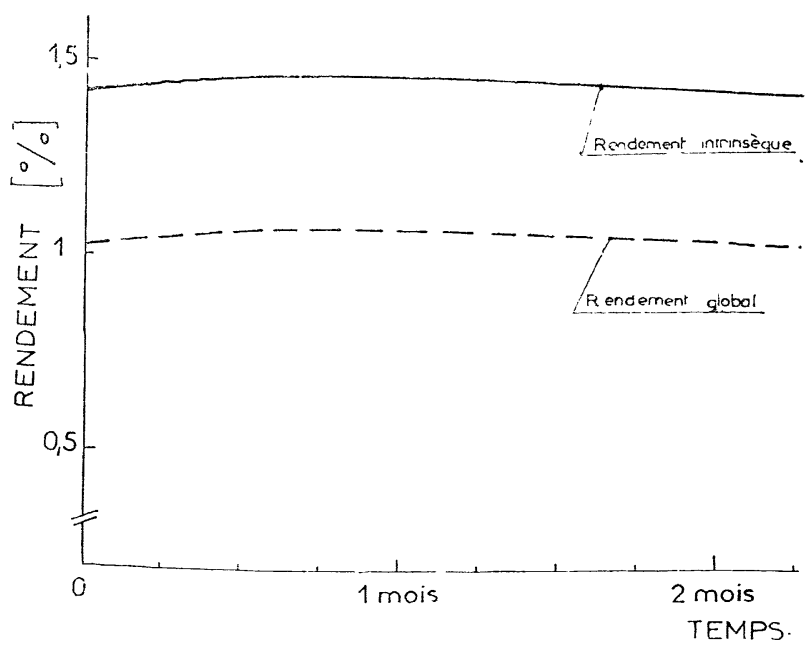

Fic. 11. - Variation du rendement des piles solaires au CdS en fonction du temps. 
suivie d'une lente décroissance. Le rendement reste donc à peu près constant pendant une période de 2 à 3 mois. Les mesures se poursuivent afin de constater s'il y a une évolution des photopiles pour des temps plus longs.

4.2.g. Puissance massique. - La puissance massique est défini par l'énergie électrique fournie par unité de masse pour une énergie lumineuse inciderıte donnée, que nous avons choisie à $140 \mathrm{~mW} / \mathrm{cm}^{2}$ correspondant au rayonnement solaire en altitude : on obtient, suivant les divers supports utilisés, une puissance de 20 à $80 \mathrm{~W} / \mathrm{kg}$.

5. Discussion. - L'effet photovoltaïque observé pourrait s'expliquer par plusieurs mécanismes simples.

$1^{\circ}$ Existence d'une homojonction n-p. - Cette hypothèse est tutalement impossible, car le cuivre utilisé peut introduire des pièges mais ne peut en aucun cas doper le CdS en sorte de le faire devenir $p$; d'ailleurs, il semble bien qu'actuellement du CdS $p$ n'ait jamais pu être obtenu à température ordinaire.

D'autre part, les réponses spectrales effectuées montrent que le maximum de l'effet est obtenu pour une longueur d'onde $(06 \mu)$ supérieure à la longueur d'onde correspondante au " gap " du $\operatorname{CdS}(0,51 \mu)$.

En outre, l'ordre de grandeur des tensions mesurées - toujours inlérieures à $0,5 \mathrm{~V}$ - n'est pas comparable à la valeur correspondant au gap du $\mathrm{CdS}$ qui est d'environ $2.4 \mathrm{eV}$.

$2^{\circ}$ Injection d'électron, métal-semiconducteur. Deux expériences infirment cette théorie dans le cas du CdS :

- Le cuivre peut être remplacé par une couche de $\mathrm{CuCl}$ sans que les caractéristiques changent de manière notable.

- L'absolue nécessité du traitement aux ions $\mathrm{Cl}^{-}$ ne se justifierait plus.

$3^{0}$ Existence d'une pseudo-jonction n-n. - Un tel modèle, bien qu'il soit possible, est impuissant à expliquer plusieurs faits observés :
- des photopiles traitées chimiquement à température ordinaire avant tout chauffage, révèlent une efficacité faible mais indubitable; or à $20^{\circ} \mathrm{C}$ aucune diffusion n'est possible et partant aucune jonction ne peut exister ;

- le milieu dans lequel s'effectue la première recuisson a une influence déterminante sur les résultats obtenus, ce qui ne devrait pas être le cas avec l'hypothèse envisagée.

Enfin, même à $300^{\circ} \mathrm{C}$, il est extrêmement peu probable que le cuivre diffuse à l'intérieur des monocristaux de CdS suffisamment pour créer une jonction.

$4^{0}$ Hétérojonction CdS (dopé $\left.n\right)-\mathrm{Cu}_{2(1-x)} \mathrm{S}$. Cette dernière hypothèse n'est en contradiction avec aucun des phénomènes observés jusqu'à présent : en particulier, toutes les influences extérieures citées (milieu ambiant et traitement chimique) peuvent facilement agir sur la formation du second composant de l'hétérojonction : de même l'existence d'un effet sans qu'il y ait eu recuisson peut s'expliquer par un processus purement chimique.

Le traitement théorique développé dans le chapitre 3 basé sur un modèle d'hétérojonction donne une base satisfaisante pour l'interprétation de l'ensemble de faits observés sur les piles solaires en couche mince de $\mathrm{CdS}$ recouvertes d'une couche semitransparente de cuivre.

Ce traitement peut être amélioré par des calculs précis de statistique de recombinaison au niveau de l'interface, ce qui donnerait une expression du courant dans une situation expérimentale donnée.

Des études expérimentales complémentaires considérant essentiellement les propriétés de surface et d'interface sont susceptibles d'apporter des données importantes pour l'élaboration de certains détails de la théorie exposée ici.

Il est évident qu'une meilleure connaissance des phénomènes d'interface contribuerait à une amélioration systématique des rendements de ce type de photopiles.

\section{BIBLIOGRAPHIE}

1] Balkanski (M.) et Chaves (Renata), J. Physique, 1966, 27, 173.

[2] Kenting (P. N.), J. Appl. Phys., 1965, 36, 564.

[3] Shockley (W.) et Read (W.), Phys. Res., 1952, 87, 835.

[4] Shockley (C.) et Shockley (W.), Phys. Rev., 1958, 109,1103
[5] Lax (M.), Phys. Rev., 1960, 119, 1502.

[6] Kröger (F.), Vink (H.) et Van der Boomgatrd, Z. Phys. Chem., 1954, B 203, 1.

[7] Duc Cuong (N.) et Blair (J.), J. Appl. Phys., 1966, 37, 1660 . 